\title{
A register-based SAH study in Japan: high incidence rate and recent decline trend based on lifestyle
}

\author{
Fusao Ikawa, MD, PhD, ${ }^{1}$ Akio Morita, MD, PhD, ${ }^{2}$ Takeo Nakayama, MD, PhD, ${ }^{3}$ \\ Yoshihito Goto, MD, MPH, ${ }^{4}$ Nobuyuki Sakai, MD, PhD, ${ }^{5}$ Koji lihara, MD, PhD, ${ }^{6}$ \\ Yoshiaki Shiokawa, MD, PhD, ${ }^{7}$ ssao Date, MD, PhD, ${ }^{8}$ Kazuhiko Nozaki, MD, PhD, ${ }^{9}$ \\ Hiroyuki Kinouchi, MD, PhD, ${ }^{10}$ Kiyohiro Houkin, MD, PhD, ${ }^{11}$ Nobuhito Saito, MD, PhD, ${ }^{12}$ \\ Teiji Tominaga, MD, PhD, ${ }^{13}$ Michiyasu Suzuki, MD, PhD, ${ }^{14}$ Susumu Miyamoto, MD, PhD, ${ }^{15}$ \\ Kaoru Kurisu, MD, PhD, ${ }^{1}$ Hajime Arai, MD, PhD, ${ }^{16}$ and the Japan Neurosurgical Society
}

1Department of Neurosurgery, Graduate School of Biomedical and Health Sciences, Hiroshima University, Hiroshima; ${ }^{2}$ Department of Neurological Surgery, Nippon Medical School Hospital, Tokyo; Departments of ${ }^{3}$ Health Informatics and ${ }^{4} \mathrm{Clinical}$ Epidemiology, School of Public Health, Graduate School of Medicine, Kyoto University, Kyoto; ${ }^{5}$ Department of Neurosurgery, Kobe City Medical Center General Hospital, Kobe; ${ }^{6}$ Department of Neurosurgery, Graduate School of Medical Sciences, Kyushu University, Fukuoka; ${ }^{7}$ Department of Neurosurgery, Kyorin University School of Medicine, Tokyo; ${ }^{8}$ Department of Neurological Surgery, Okayama University Graduate School of Medicine, Okayama; ${ }^{9}$ Department of Neurosurgery, Shiga University of Medical Science, Shiga; ${ }^{10}$ Department of Neurosurgery, University of Yamanashi; ${ }^{11}$ Department of Neurosurgery, Hokkaido University Graduate School of Medicine, Sapporo; ${ }^{12}$ Department of Neurosurgery, The University of Tokyo; ${ }^{13}$ Department of Neurosurgery, Tohoku University Graduate School of Medicine, Sendai; ${ }^{14}$ Department of Neurosurgery, Yamaguchi University School of Medicine, Ube; ${ }^{15}$ Department of Neurosurgery, Kyoto University Graduate School of Medicine, Kyoto; and ${ }^{16}$ Department of Neurosurgery, Juntendo University, Tokyo, Japan

OBJECTIVE Japan has been reported to have the highest (and increasing) incidence of subarachnoid hemorrhage $(\mathrm{SAH})$ in the world. However, there has never been a report on the nationwide incidence rate and recent trends for SAH in Japan. In this register-based study, the authors aimed to clarify the estimated nationwide SAH incidence rate and the recent trend in SAH incidence in Japan and the reasons for any changes in this trend.

METHODS The authors compiled data from the Japanese Ministry of Health, Labour and Welfare and from the records of the Japan Neurosurgical Society. They reviewed the age-standardized nationwide SAH mortality rate, the estimated age-standardized SAH incidence rate according to the age-standardized SAH mortality rate, and the estimated crude SAH incidence rate, including the $95 \%$ confidence intervals, from 2003 to 2015 . The trends in the number of treatments for unruptured and ruptured cerebral aneurysms, as well as the prevalence of hypertension, current smoking status, and use of cholesterol-lowering drugs, were assessed. The estimated treatment rate for unruptured cerebral aneurysms (UCAs) was also calculated along with the $95 \%$ confidence interval.

RESULTS The estimated age-standardized nationwide SAH incidence rate significantly declined from 31.34 cases (95\% Cl 31.32-31.34) to 27.63 (95\% Cl 27.59-27.63; decrease by 11.8\%) per 100,000 person-years. This decline was based on the 2010 population as the reference from 2003 through 2015 and a case-fatality rate of SAH that was assumed to decrease by $1 \%$ annually from $44 \%$ in 2003 to $32 \%$ in 2015 . According to sensitivity analysis, the change rate of the estimated age-standardized SAH incidence rate ranged from $-56.69 \%$ to $23.27 \%$, with a mean of $-30.91 \%$ (SD $20.52 \%$ ), and there were decline trends in $92 \%$ of all trends. The estimated nationwide crude SAH incidence rate also showed a significant decline from 23.79 (95\% Cl 23.78-23.79) to 20.25 (95\% Cl 20.24-20.25; decrease by $14.9 \%)$ per 100,000 person-years. The trend in treatment numbers for UCAs increased significantly $(p<0.0001)$ from 2003 through 2015; however, the estimated treatment rate for UCAs was only $0.19(95 \% \mathrm{Cl} 0.19-0.20)$ to $0.51(95 \% \mathrm{Cl} 0.50-0.51)$ among all UCA patients. The prevalence of hypertension (males, $p=0.0003$; females, $p<0.0001$ ) and current smoking

ABBREVIATIONS JNS = Japan Neurosurgical Society; MHLW = Ministry of Health, Labour and Welfare; NHNS = National Health and Nutrition Survey; RCA = ruptured cerebral aneurysm; $\mathrm{SAH}=$ subarachnoid hemorrhage; $\mathrm{UCA}=$ unruptured cerebral aneurysm.

SUBMITTED October 29, 2019. ACCEPTED January 13, 2020.

INCLUDE WHEN CITING Published online March 27, 2020; DOI: 10.3171/2020.1.JNS192848. 
status (males, $p<0.0001$; females, $p=0.0002$ ) declined significantly from 2003 through 2015, while the use of cholesterol-lowering drugs increased significantly (males, $p<0.0001$; females, $p=0.0005$ ) during the same period.

CONCLUSIONS The estimated nationwide SAH incidence rate in Japan was higher than rates in other countries, although it has declined recently. An improving lifestyle may have contributed to the declining rate of SAH incidence in Japan.

https://thejns.org/doi/abs/10.3171/2020.1.JNS192848

KEYWORDS subarachnoid hemorrhage; epidemiology; incidence; trends; registry; vascular disorders

$\mathrm{F}$ INLAND $^{1}$ and $\operatorname{Japan}^{2}$ have been reported to have the highest incidences of subarachnoid hemorrhage (SAH) in the world. However, recently available evidence does not support a high incidence in Finland ${ }^{3}$ because the evidence was based on regional studies without standardization of reference populations ${ }^{4-6}$ and because, according to other data, the incidence rate decreased by 24\% between 1998 and 2012 along with a simultaneous decrease in smoking prevalence. ? According to a recent meta-analysis, ${ }^{8}$ the crude global incidence of SAH has declined from 10.2 per 100,000 person-years in 1980 to 6.1 in 2010 (decline by 40.2\%), paralleling a global decrease in mean blood pressure and smoking prevalence. On the other hand, the regional incidence rate of SAH in Japan was reported as 13.7 per 100,000 person-years in $1977^{9}$ and 27.9 per 100,000 person-years in $2006,{ }^{10}$ and according to the aforementioned meta-analysis, ${ }^{8}$ there was a significant increase in the crude SAH incidence and sex-adjusted SAH incidence (by 59.1\%) over the past 3 decades. However, the Japanese data used in this study were from up to 2008 and all studies were regional rather than nationwide. Based on data to date from the Ministry of Health, Labour and Welfare (MHLW) in Japan, the recent crude SAH mortality has declined after a peak in 2005 , even though the peak in the population occurred in 2008. ${ }^{11}$

In contrast, the number of treatments for unruptured cerebral aneurysms (UCAs) has been increasing in Japan because UCAs are frequently discovered at screening checkups. ${ }^{12}$ Furthermore, the high rates of total UCA treatments $(45.5 \%)$ and small UCA $(<5 \mathrm{~mm}$ in diameter) treatments $(37.1 \%)^{13,14}$ are attributable to a 2.8 times higher rupture rate of UCAs in Japan than in Western countries. ${ }^{15}$ However, there is no evidence that the increasing trend in the number of treatments for UCAs or changing lifestyle habits would contribute to reducing the incidence of SAH in Japan. Therefore, in this register-based study, we aimed to clarify the estimated nationwide SAH incidence rate and the recent trend in SAH incidence in Japan, as well as to investigate the effects of the increasing number of treatments for UCAs and changes in the prevalence of hypertension, current smoking, and use of cholesterol-lowering drugs on this trend.

\section{Methods}

\section{Protocol Approval, Patient Consent, Role of the Funding Source, and Data Availability}

This study was approved by the Institutional Review Board of Hiroshima University and the Japan Neurosurgical Society (JNS; clinical trial registration no.: UMIN000034241, http://www.umin.ac.jp/icdr/index.html).
Because of the anonymous nature of the data, the requirement for informed consent by patients was waived, and an opt-out method was used as the informed consent for this study.

The anonymized data for this study will be shared by the request of any qualified investigator to the corresponding author. Investigators may access the data in the MHLW through the Statistics link on the website of the Japan MHLW. Primary data from the JNS will be made available upon reasonable request in accordance with the review board of the JNS.

Funders of the study had no role in the study design, data collection, data analysis, data interpretation, or writing of this report. The corresponding author has full access to all data in the study and has final responsibility for the decision to submit for publication.

\section{Data Extraction}

We extracted data from records in a database at the MHLW in Japan. Since 1970, the MHLW has conducted surveys every 5 years, the same years as the general population census. ${ }^{16}$ Every survey period lasted for the 12 months of the fiscal year (April 1 of the survey year to March 31 of the next year). The MHLW approved the secondary use of the data, which included the total population and the crude number of deaths caused by SAH every 5 years in males and females. ${ }^{17}$ Using these data and a direct method, we calculated the standardized nationwide SAH mortality rate per 100,000 person-years by age and sex using the years 2005 and 2010 as representative samples $^{16}$ between 2003 and 2015. From the database of the MHLW, we extracted annual trends in the age-standardized percentage of subjects with high systolic blood pressure $(>140 \mathrm{~mm} \mathrm{Hg})$, current smoking status, and use of cholesterol-lowering drugs between 2003 and 2015. ${ }^{18}$ Additionally, we reviewed data from the JNS for 2003-2015. The JNS requires a certain number of operations to certify training programs and receives reports from each facility annually to meet this requirement. These reports include the number of UCAs and ruptured cerebral aneurysms (RCAs) treated with surgical clipping or endovascular coiling (radical treatment). We extracted these annual data for each procedure from 2003 to 2015 with the approval of the JNS.

\section{Accuracy of Register-Based Diagnosis of SAH, Mortality, and MHLW Data}

As required by law, all causes of death were reported to the government office. Emergent or autopsy CT imaging was conducted for almost all sudden-death patients in 
Japan. The Organisation for Economic Co-operation and Development data show that Japan has the most acute care beds per 1000 population and the largest number of CT units per million population under a universal healthcare system. ${ }^{19}$ Additionally, the MHLW conducted a National Health and Nutrition Survey (NHNS) to determine levels of health and nutrition in Japan..$^{20,21}$ The NHNS is a national nutrition survey conducted annually since 1945 by public health centers under the supervision of the MHLW in accordance with the Health Promotion Law. The NHNS has been detailed elsewhere ${ }^{18,20}$ Briefly, based on the population census, 475 of the approximately 1 million census units were randomly sampled as survey areas. All noninstitutionalized Japanese persons aged 1 year or older and living in survey areas were asked to participate, excluding individuals holding foreign citizenship, those whose diet was not self-selected, and those using a special diet (mainly due to disease). By using the NHNS, the trends in hypertension, current smoking status, and use of cholesterol-lowering drugs from 2003 to 2015 were researched. Unfortunately, data on the use of cholesterollowering drugs started in 2005; therefore, the data from 2003 to 2004 were not available.

The accuracy of register-based diagnoses of SAH in the JNS is the responsibility of the director of each institute and is regularly verified by the JNS. All definitive diagnoses and medical records are linked to the universal medical insurance system and regularly checked by the MHLW. The JNS data include all major facilities listed in the Appendix and certified facilities belonging to the major facilities in Japan and all cases of aneurysm treatment in these facilities.

\section{Calculation of SAH Mortality Rate and Incidence Rate}

The SAH mortality rate and incidence rate are expressed as cases per 100,000 person-years. First, from the statistics of the MHLW, we collected the crude number of deaths caused by SAH every 5 years for males and females. ${ }^{17}$ Additionally, we retrieved the 5-year age group numbers from census populations in 2005 and $2010 . .^{16}$ Therefore, the SAH mortality rate could be standardized for age and sex by using the 2005 and 2010 populations as the standard. ${ }^{16}$ The age-standardized SAH mortality rate and $95 \%$ confidence interval could be calculated using the age-specific SAH mortality rates for each 5-year age group, dividing the number of deaths by the respective population and then multiplying the result by 100,000 person-years. The estimated age-standardized SAH incidence rate per 100,000 person-years could be estimated according to the age-standardized SAH mortality rate. The casefatality rate of SAH has been reported to be from $32 \%$ to $45 \%$-improving but higher in the elderly. ${ }^{22-26}$ Therefore, the case-fatality rate of SAH was assumed to decrease by $1 \%$ annually from $44 \%$ in 2003 to $32 \%$ in 2015 (Table 1). Additionally, we conducted a sensitivity analysis by changing both the case-fatality rate of SAH and the annual change in the case-fatality rate of SAH based on a range of assumed mortality rates and the annual change rate. The case-fatality rate of SAH was changed from $25 \%$ to $45 \%$ at 5\% intervals, and the annual change in the case-fatality rate of SAH changed from $-1 \%$ to $1 \%$ at $0.5 \%$ intervals

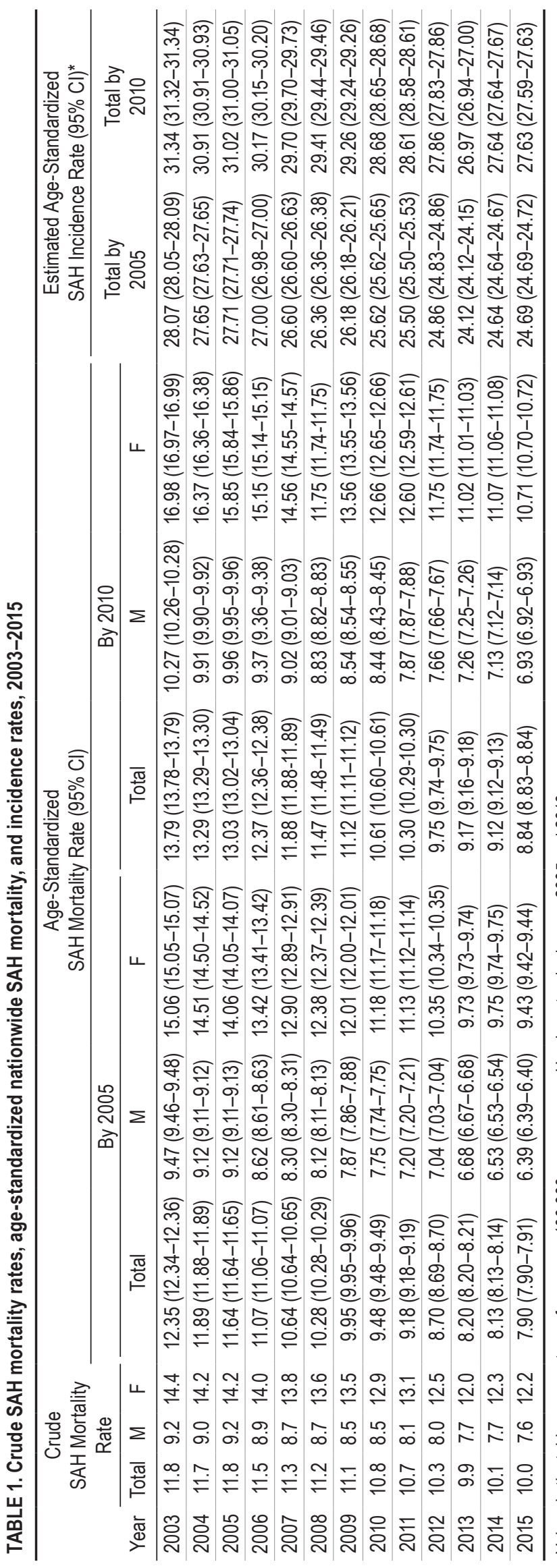

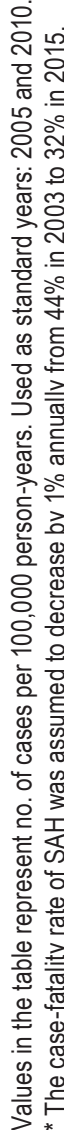


TABLE 2. Sensitivity analysis by the case-fatality rate of SAH and the annual change, 2003-2015

\begin{tabular}{|c|c|c|c|c|c|}
\hline \multirow{2}{*}{$\begin{array}{l}\text { Case-Fatality } \\
\text { Rate of SAH }\end{array}$} & \multicolumn{5}{|c|}{ Annual Change in Case-Fatality Rate of SAH from 2003 to 2015} \\
\hline & $1 \%$ & $0.5 \%$ & 0 & $-0.5 \%$ & $-1 \%$ \\
\hline \multicolumn{6}{|l|}{$45 \%$} \\
\hline 2003 & $30.64(30.62-30.64)$ & $30.64(30.62-30.64)$ & $30.64(30.62-30.64)$ & $30.64(30.62-30.64)$ & $30.64(30.62-30.64)$ \\
\hline 2015 & $15.51(15.49-15.51)$ & $17.33(17.31-17.33)$ & $19.64(19.62-19.64)$ & $22.67(22.64-22.67)$ & $26.79(26.76-26.79)$ \\
\hline $\begin{array}{l}\text { Change rate from } \\
2003 \text { to } 2015(\%)\end{array}$ & -49.38 & -43.44 & -35.9 & -26.01 & -12.57 \\
\hline \multicolumn{6}{|l|}{$40 \%$} \\
\hline 2003 & $34.48(34.45-34.48)$ & $34.48(34.45-34.48)$ & $34.48(34.45-34.48)$ & $34.48(34.45-34.48)$ & $34.48(34.45-34.48)$ \\
\hline 2015 & $17.00(16.98-17.00)$ & $19.22(19.20-19.22)$ & $22.10(22.08-22.10)$ & $26.00(25.97-26.00)$ & $31.57(31.54-31.57)$ \\
\hline $\begin{array}{l}\text { Change rate from } \\
2003 \text { to } 2015(\%)\end{array}$ & -50.70 & -44.26 & -35.90 & -24.59 & -8.44 \\
\hline \multicolumn{6}{|l|}{$35 \%$} \\
\hline 2003 & $39.40(39.37-39.40)$ & $39.40(39.37-39.40)$ & $39.40(39.37-39.40)$ & $39.40(39.37-39.40)$ & $39.40(39.37-39.40)$ \\
\hline 2015 & 18.81 (18.79-18.81) & $21.56(21.54-21.56)$ & $25.26(25.23-25.26)$ & $30.48(30.45-30.48)$ & $38.43(38.39-38.43)$ \\
\hline $\begin{array}{l}\text { Change rate from } \\
2003 \text { to } 2015(\%)\end{array}$ & -52.26 & -45.28 & -35.89 & -22.64 & -2.46 \\
\hline \multicolumn{6}{|l|}{$30 \%$} \\
\hline 2003 & $45.97(45.93-45.97)$ & $45.97(45.93-45.97)$ & $45.97(45.93-45.97)$ & $45.97(45.93-45.97)$ & $45.97(45.93-45.97)$ \\
\hline 2015 & $21.05(21.02-21.05)$ & $24.56(24.53-24.56)$ & $29.47(29.43-29.47)$ & $36.83(36.79-36.83)$ & $49.11(49.06-49.11)$ \\
\hline $\begin{array}{l}\text { Change rate from } \\
2003 \text { to } 2015(\%)\end{array}$ & -54.21 & -46.57 & -35.89 & -19.88 & 6.83 \\
\hline \multicolumn{6}{|l|}{$25 \%$} \\
\hline 2003 & $55.16(55.12-55.16)$ & $55.16(55.12-55.16)$ & $55.16(55.12-55.16)$ & $55.16(55.12-55.16)$ & $55.16(55.12-55.16)$ \\
\hline 2015 & $23.89(23.86-23.89)$ & $28.52(28.48-28.52)$ & $35.36(35.32-35.36)$ & $46.53(46.47-46.53)$ & $68.00(67.92-68.00)$ \\
\hline $\begin{array}{l}\text { Change rate from } \\
2003 \text { to } 2015(\%)\end{array}$ & -56.69 & -48.30 & -35.90 & -15.65 & 23.27 \\
\hline
\end{tabular}

Values in the table body represent the estimated age-standardized SAH incidence rate per 100,000 person-years in total and the change rate (\%) from 2003 to 2015.

(Table 2). The formula for the estimated age-standardized $\mathrm{SAH}$ incidence rate was the age-standardized SAH mortality rate/(case-fatality rate of $\mathrm{SAH} / 100)$.

Second, the estimated crude SAH incidence rate was calculated using the number of radical treatments of RCAs from the JNS registered data and the crude number of deaths caused by SAH from the MHLW. The total number of SAHs per year was calculated with the following formula: number of deaths caused by $\mathrm{SAH}+$ number of RCA survivors after radical treatment + number of conservative treatment survivors after SAH. The number of RCA survivors after radical treatment was calculated with the following formula: number of radical treatments for RCA $\times(1-[10 \%-20 \%] / 100)$. We used this formula because the case-fatality rate after radical treatment in Japan has been reported to range from $10 \%$ to $20 \%,{ }^{27,28}$ with the assumption of no change in the case-fatality rate after radical treatment during the study period. The number of conservative treatment survivors after SAH $(\alpha)$ and the SAH incidence rate of conservative treatment survivors $(\beta)$ were assessed. The estimated crude SAH incidence rate was (total number of SAHs in a year/population in the same year) $\times 100,000$. Finally, total number of SAHs $-\alpha$ $=$ number of deaths caused by SAH + (number of radical treatments for RCA $) \times(1-[10 \%-20 \%] / 100)$, and crude
SAH incidence rate $-\beta$ per 100,000 person-years $=($ total number of SAHs $-\alpha)$ /population in the same year. Therefore, we can estimate the crude $\mathrm{SAH}$ incidence rate $-\beta$ per 100,000 person-years including the $95 \%$ confidence interval (Table 3).

\section{Estimation of Treatment Rate for UCAs}

To estimate the treatment rate for UCAs, we used a range from approximately $3 \%$ to $5 \%$ for the prevalence rate of UCAs in patients 50 years of age. ${ }^{29}$ The estimated number of patients with UCAs and the estimated treatment rate for UCAs per year could be calculated based on the following formula. The estimated number of patients with UCAs per year was the number of people over 15 years of age $\times[(3 \%-5 \%) / 100]$ because the incidence of de novo UCAs was $0.6 \%$ per patient-year and negligible. ${ }^{30}$ The estimated treatment rate for UCAs per year including the $95 \%$ confidence interval was (number of treatments of UCAs/estimated number of patients with UCAs) $\times 100$ (Table 4). In this formula, we assumed that multiple UCAs were treated simultaneously and all de novo aneurysms were included among UCAs.

\section{Statistical Analysis}

We used Stata statistical software (version 13.1, Stata 
TABLE 3. Estimation of the nationwide crude SAH incidence rate from 2003 to 2015

\begin{tabular}{|c|c|c|c|c|c|c|c|c|c|c|}
\hline \multirow[b]{2}{*}{ Year } & \multirow{2}{*}{$\begin{array}{c}\text { No. of Deaths } \\
\text { Caused by } \\
\text { SAH }\end{array}$} & \multirow{2}{*}{$\begin{array}{c}\text { No. of } \\
\text { Radical Txs } \\
\text { for RCAs }\end{array}$} & \multicolumn{2}{|c|}{$\begin{array}{l}\text { No. of RCA Survivors } \\
\text { After Radical Tx }\end{array}$} & \multirow{2}{*}{$\begin{array}{c}\alpha \\
\text { After } \\
\text { SAH }\end{array}$} & \multicolumn{2}{|c|}{$\begin{array}{l}\text { Estimated Total No. } \\
\text { of SAHs }-\alpha\end{array}$} & \multirow{2}{*}{$\begin{array}{c}\text { Population } \\
(\times 1000)\end{array}$} & \multicolumn{2}{|c|}{$\begin{array}{l}\text { Estimated Crude SAH Incidence Rate - } \\
\quad \beta / 100,000 \text { Person-Yrs (95\% Cl) }\end{array}$} \\
\hline & & & $10 \%$ CFR & $20 \%$ CFR & & $10 \%$ CFR & $20 \%$ CFR & & $10 \%$ CFR & $20 \%$ CFR \\
\hline 2003 & 14,929 & 19,305 & 17,375 & 15,444 & $\alpha 1$ & 30,373 & & 127,694 & $25.30(25.29-25.31)$ & 23.79 (23.78-23.79) \\
\hline 2004 & 14,737 & 19,366 & 17,429 & 15,493 & $\alpha 2$ & 30,230 & 32,166 & 127,787 & $25.17(25.16-25.18)$ & $23.66(23.65-23.66)$ \\
\hline 2005 & 14,883 & 18,942 & 17,048 & 15,154 & $\alpha 3$ & 30,037 & 31,931 & 127,768 & $24.99(24.98-25.00)$ & $23.51(23.50-23.52)$ \\
\hline 2006 & 14,466 & 19,097 & 17,187 & 15,278 & $\alpha 4$ & 29,744 & 53 & 127,901 & $24.75(24.74-24.76)$ & $23.26(23.25-23.26)$ \\
\hline 2007 & 14,243 & 19,085 & 17,177 & 15,268 & $\alpha 5$ & 29,511 & 31,420 & 128,033 & $24.54(24.53-24.55)$ & $23.05(23.04-23.06)$ \\
\hline 2008 & 14,075 & 18,815 & 16,934 & 15,052 & $\alpha 6$ & 29,127 & 31,009 & 128,084 & $24.21(24.20-24.22)$ & $22.74(22.73-22.75)$ \\
\hline 2009 & 13,923 & 18,758 & 16,882 & 15,006 & $\alpha 7$ & 28,929 & 30,805 & 128,032 & $24.06(24.05-24.07)$ & $22.60(22.59-22.60)$ \\
\hline 2010 & 13,591 & 18,047 & 16,242 & 14,438 & $\alpha 8$ & 28,029 & 29,833 & 128,057 & $23.30(23.29-23.30)$ & $21.89(21.88-21.90)$ \\
\hline 2011 & 13,460 & 17,616 & 15,854 & 14,093 & $\alpha 9$ & 27,553 & 29,314 & 127,799 & $22.94(22.93-22.95)$ & $21.56(21.55-21.57)$ \\
\hline 2012 & 13,004 & 17,618 & 15,856 & 14,094 & $\alpha 10$ & 27,098 & 28,860 & 127,515 & $22.63(22.62-22.64)$ & $21.25(21.24-21.26)$ \\
\hline 2013 & 12,479 & 17,040 & 15,336 & 13,632 & $\alpha 11$ & 26,111 & 27,815 & 127,298 & $21.85(21.84-21.86)$ & $20.51(20.50-20.52)$ \\
\hline 2014 & 12,662 & 16,751 & 15,076 & 13,401 & $\alpha 12$ & 26,063 & 27,738 & 127,083 & $21.83(21.82-21.83)$ & $20.51(20.50-20.52)$ \\
\hline 2015 & 12,476 & 16,571 & 14,914 & 13,257 & $\alpha 13$ & 25,733 & 27,390 & 127,095 & $21.55(21.54-21.56)$ & $20.25(20.24-20.25)$ \\
\hline
\end{tabular}

$\alpha=$ number of conservative treatment survivors; $\beta=$ incidence rate of conservative treatment survivors per 100,000 person-years; CFR $=$ case-fatality rate after radical treatment; Tx = treatment.

Corp) for analyses. By using the linear regression model to compute the slope, we examined several trends from 2003 to 2015: trends in the nationwide age-standardized SAH mortality rate, crude SAH incidence rate, treatment numbers for UCAs and RCAs, and number of deaths caused by $\mathrm{SAH}$, as well as annual trends in the age-standardized percentage of subjects with systolic blood pressure over 140 $\mathrm{mm} \mathrm{Hg}$, current smokers, and patients taking cholesterollowering drugs.

\section{Results}

Table 1 shows the crude SAH mortality rates, agestandardized nationwide SAH mortality rates, and inci- dence rates per 100,000 person-years from 2003 to 2015 using 2005 and 2010 as standard years. The crude SAH mortality rate significantly declined from 11.8 to 10.0 (by $15.3 \%$ ) per 100,000 person-years during this period ( $\mathrm{p}<0.001)$. The age-standardized SAH mortality rates from 2003 to 2015 also significantly declined from 12.35 (95\% CI 12.34-12.36) to 7.90 (95\% CI 7.90-7.91; decrease by $37.0 \%$, p < 0.001) per 100,000 person-years using the 2005 Japanese population as standard and from 13.79 (95\% CI 13.78-13.79) to 8.84 (95\% CI 8.83-8.84; decrease by $35.9 \%$, p < 0.001$)$ per 100,000 person-years using the 2010 Japanese population as standard. The estimated age-standardized nationwide $\mathrm{SAH}$ incidence rate also declined from 28.07 (95\% CI 28.05-28.09) to 24.69

TABLE 4. Estimation of treatment rate for UCAs from 2003 to 2015

\begin{tabular}{|c|c|c|c|c|c|c|}
\hline \multirow[b]{2}{*}{ Year } & \multirow{2}{*}{$\begin{array}{l}\text { No. of Population } \\
\text { Over } 15 \text { Yrs } \\
(\times 1000)\end{array}$} & \multicolumn{2}{|c|}{ Estimated No. of UCA Patients $\times 1000$} & \multirow[b]{2}{*}{$\begin{array}{l}\text { Tx No. for } \\
\text { UCAs }\end{array}$} & \multicolumn{2}{|c|}{ Estimated Tx Rate for UCAs per Yr Expressed as \% (95\% Cl) } \\
\hline & & $\begin{array}{c}3 \% \text { Prevalence } \\
\text { of UCAs }\end{array}$ & $\begin{array}{c}5 \% \text { Prevalence } \\
\text { of UCAs }\end{array}$ & & $\begin{array}{c}3 \% \text { Prevalence } \\
\text { of UCAs }\end{array}$ & $\begin{array}{c}5 \% \text { Prevalence } \\
\text { of UCAs }\end{array}$ \\
\hline 2003 & 109,715 & 3,291 & 5,486 & 10,666 & $0.32(0.32-0.33)$ & $0.19(0.19-0.20)$ \\
\hline 2004 & 109,953 & 3,299 & 5,498 & 10,607 & $0.32(0.32-0.33)$ & $0.19(0.19-0.20)$ \\
\hline 2005 & 110,183 & 3,305 & 5,509 & 11,132 & $0.34(0.33-0.34)$ & $0.20(0.20-0.21)$ \\
\hline 2006 & 110,326 & 3,310 & 5,516 & 11,892 & $0.36(0.35-0.37)$ & $0.22(0.21-0.22)$ \\
\hline 2007 & 110,456 & 3,314 & 5,523 & 13,165 & $0.40(0.39-0.40)$ & $0.24(0.23-0.24)$ \\
\hline 2008 & 110,545 & 3,316 & 5,527 & 14,025 & $0.42(0.42-0.43)$ & $0.25(0.25-0.26)$ \\
\hline 2009 & 110,631 & 3,319 & 5,532 & 14,135 & $0.43(0.42-0.43)$ & $0.26(0.25-0.26)$ \\
\hline 2010 & 111,254 & 3,338 & 5,563 & 16,008 & $0.48(0.47-0.49)$ & $0.29(0.28-0.29)$ \\
\hline 2011 & 111,094 & 3,333 & 5,555 & 15,830 & $0.47(0.47-0.48)$ & $0.28(0.28-0.29)$ \\
\hline 2012 & 110,968 & 3,329 & 5,548 & 16,198 & $0.49(0.48-0.49)$ & $0.29(0.29-0.30)$ \\
\hline 2013 & 110,908 & 3,327 & 5,545 & 16,840 & $0.51(0.50-0.51)$ & $0.30(0.30-0.31)$ \\
\hline 2014 & 110,850 & 3,326 & 5,543 & 16,416 & $0.49(0.49-0.50)$ & $0.30(0.29-0.30)$ \\
\hline 2015 & 111,208 & 3,336 & 5,560 & 16,291 & $0.49(0.48-0.50)$ & $0.29(0.29-0.30)$ \\
\hline
\end{tabular}




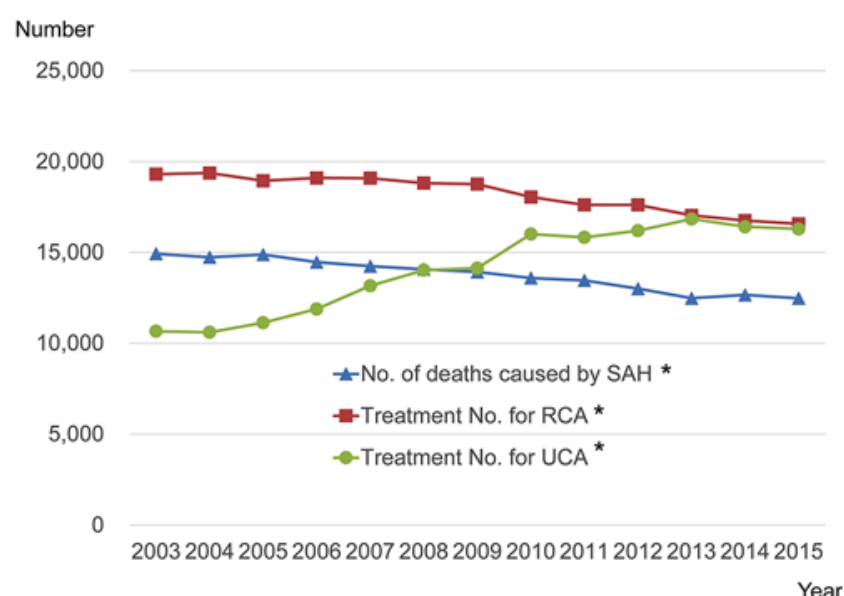

FIG. 1. Trends in treatment numbers for UCAs and RCAs and number of deaths caused by SAH in Japan, from 2003 through 2015. ${ }^{*} p<0.0001$. Figure is available in color online only.

(95\% CI 24.69-24.72; decrease by $12.0 \%$, p < 0.001) per 100,000 person-years using the 2005 population as standard and from 31.34 (95\% CI 31.32-31.34) to 27.63 (95\% CI 27.59-27.63; decrease by $11.8 \%$, p < 0.001) per 100,000 person-years using the 2010 population as standard, assuming that the case-fatality rate of SAH decreased by $1 \%$ annually from $44 \%$ in 2003 to $32 \%$ in 2015 . Table 2 shows that the change rate of the estimated age-standardized SAH incidence rate per 100,000 person-years in total SAH from 2003 to 2015 ranged from $-56.69 \%$ to $23.27 \%$, with a mean of $-30.91 \%$ (SD $20.52 \%$ ), and there were decline trends in $92 \%$ of all trends.

Table 3 reveals the number of deaths caused by $\mathrm{SAH}$, the number of radical treatments for RCAs, the number of RCA survivors after radical treatment, the number of conservative treatment survivors after $\mathrm{SAH}(\alpha)$, the estimated total number of SAHs excluding the conservative treatment survivors after $\mathrm{SAH}(\alpha)$, the population, and the estimated crude SAH incidence rate excluding the incidence rate of conservative treatment survivors after SAH ( $\beta$ ) per 100,000 person-years from 2003 to 2015. The estimated crude SAH incidence rate excluding the incidence rate of conservative treatment survivors after $\mathrm{SAH}(\beta)$ per 100,000 person-years significantly declined from 25.30 (95\% CI 25.29-25.31) to 21.55 (95\% CI 21.54-21.56; decrease by $14.8 \%$, p < 0.001$)$ per 100,000 population and from 23.79 (95\% CI 23.78-23.79) to 20.25 (95\% CI 20.2420.25 ; decrease by $14.9 \%, \mathrm{p}<0.001)$ per 100,000 personyears, if the case-fatality rate after radical treatment of SAH was $10 \%$ or $20 \%$, respectively.

Figure 1 shows the trends in radical treatment numbers for UCAs and RCAs and number of deaths caused by SAH, from 2003 through 2015. The radical treatment numbers for UCAs significantly increased ( $p<0.0001)$, whereas radical treatment numbers for RCAs and number of deaths caused by SAH significantly declined $(\mathrm{p}<$ $0.0001)$ during this period. Table 4 shows the trends in the population number over 15 years of age, the estimated number of UCA patients, the treatment number for UCAs, and the estimated treatment rate for UCAs, from 2003 to

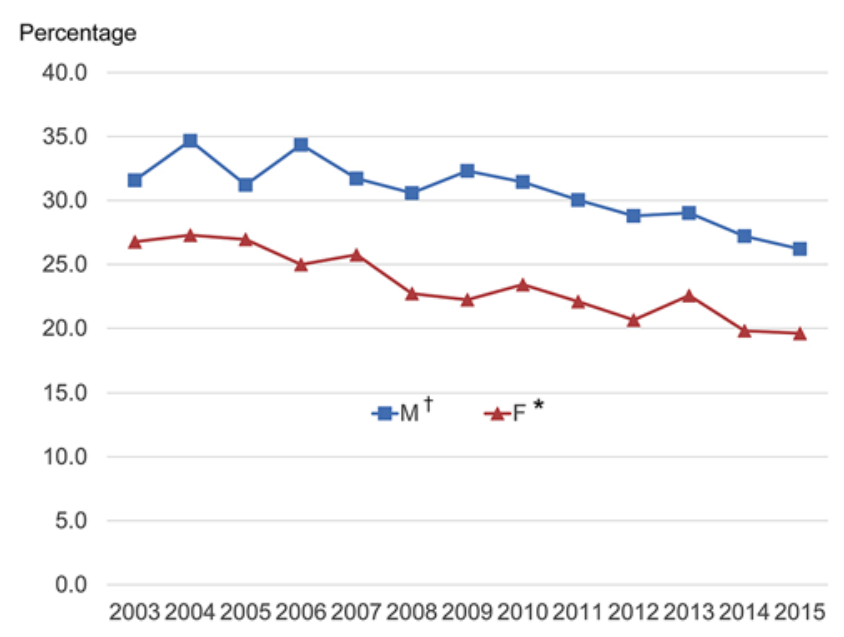

Year

FIG. 2. Annual trends in the age-standardized percentage of subjects with systolic blood pressure over $140 \mathrm{~mm} \mathrm{Hg}$. †Male $(p=0.0003)$; ${ }^{*}$ female $(p<0.0001)$. Figure is available in color online only.

2015. The estimated treatment rate for UCAs ranged from $0.32 \%(95 \%$ CI $0.32 \%-0.33 \%)$ to $0.51 \%(0.50 \%-0.51 \%)$ and $0.19 \%(0.19 \%-0.20 \%)$ to $0.30 \%(0.30 \%-0.31 \%)$, if the prevalence of UCAs was $3 \%$ or $5 \%$, respectively. Figures 2-4 show annual trends from 2003 through 2015 in the age-standardized percentage of subjects with high systolic blood pressure (over $140 \mathrm{~mm} \mathrm{Hg}$; Fig. 2), current smoking status (Fig. 3), and patients taking cholesterol-lowering drugs (Fig. 4). The trend in the percentage of males $(\mathrm{p}=0.0003)$ and females $(\mathrm{p}<0.0001)$ with high systolic blood pressure and males $(\mathrm{p}<0.0001)$ and females $(\mathrm{p}=$ 0.0002 ) currently smoking significantly declined from 2003 through 2015. The trend in the percentage of males $(\mathrm{p}<0.0001)$ and females $(\mathrm{p}=0.0005)$ taking cholesterollowering drugs significantly increased from 2005 through 2015. (Data on the use of cholesterol-lowering drugs for 2003-2004 were not available.)

\section{Discussion}

\section{Nationwide SAH Incidence Rate and Recent Trend in Japan}

To our knowledge, this is the first register-based study to report the nationwide $\mathrm{SAH}$ incidence rate and the recent trend in SAH in Japan. We analyzed the estimated nationwide SAH incidence rate in Japan by using two different methods, namely the estimated age-standardized SAH incidence rate per 100,000 person-years according to MHLW data and the estimated crude SAH incidence rate excluding the incidence rate of conservative treatment survivors after SAH per 100,000 person-years according to JNS register-based data. The estimated age-standardized SAH incidence rate was higher than the estimated crude SAH incidence rate excluding the incidence rate of conservative treatment survivors after SAH. The treatment method, emergency transport rate, and emergent imaging rate for $\mathrm{SAH}$ have never changed rapidly; however, aging has accelerated during the study period in Japan. This rapid aging could be the reason for the difference between the 


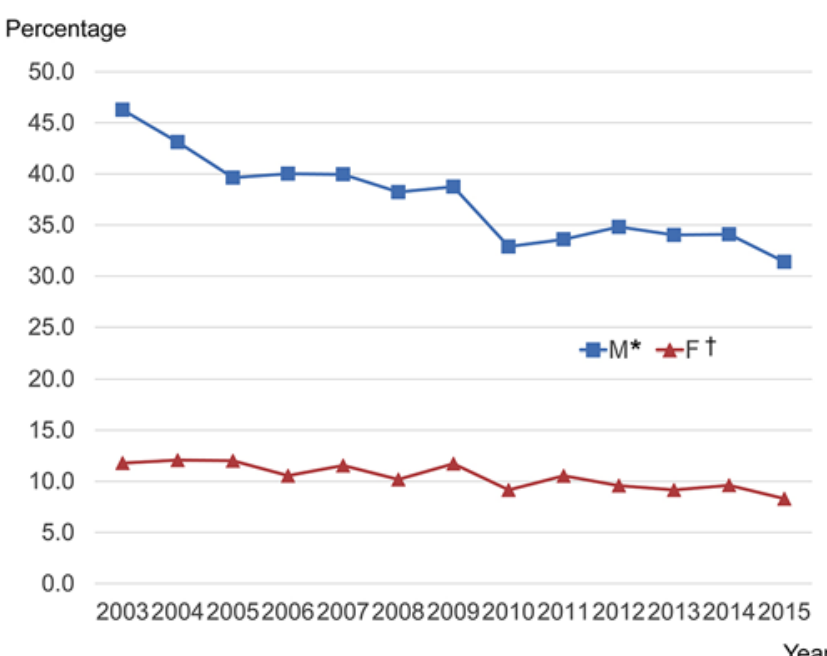

FIG. 3. Annual trends in the age-standardized percentage of subjects with current smoking status. ${ }^{*}$ Male $(p<0.0001)$; †female $(p=0.0002)$. Figure is available in color online only.

two estimated nationwide SAH incidence rates. We found a higher decline in the SAH mortality rate after age standardization than in the crude data (Table 1). Additionally, there was no longer a significant increase in the SAH incidence rate after age standardization by meta-analysis. ${ }^{8} \mathrm{We}$ selected the maximum conceivable reduction rate of the case fatality of SAH as $1 \%$ annually, from $44 \%$ in 2003 to $32 \%$ in 2015 . According to our sensitivity analysis (Table 2), there were significant decline trends in $92 \%$ of all trends. The change rate of the estimated age-standardized SAH incidence rate increased in only two assumptions from $30 \%$ and $25 \%$ with an annual $1 \%$ decline trend in the case-fatality rate of SAH. However, these two assumptions were far from real clinical evidence. Therefore, we could conclude that there was at least a significant decline in the SAH incidence rate between 2003 and 2015 in Japan and a higher SAH incidence rate in Japan compared to that of other countries. $^{8}$

\section{Reasons for a Decline in the Incidence of SAH}

We tried to verify whether the rapidly increasing trend in the treatment number for UCAs contributed to the decline in the incidence of SAH. The estimated treatment rate for UCAs from 2003 to 2015 ranged from only $0.19 \%$ to $0.51 \%$ (Table 4). The number needed to treat was 204 to 548 UCAs for 1 SAH reduction if the annual rupture rate was $0.96 \%$ per year..$^{14}$ From this fact, we could conclude that the decline in the incidence of SAH according to the increase in treating UCAs was too low.

Hypertension and smoking have been strong risk factors for $\mathrm{SAH},{ }^{31,32}$ and the use of statin drugs has been an inverse risk for SAH. ${ }^{33}$ In Japan, statins are used as the cholesterol-lowering drug in more than $80 \%$ of patients. Therefore, we investigated the trends in these lifestyle diseases and the use of cholesterol-lowering drugs. The prevalence of hypertension and smoking has declined significantly from 2003 through 2015, and the use of cholesterol-lowering drugs has increased significantly from 2005

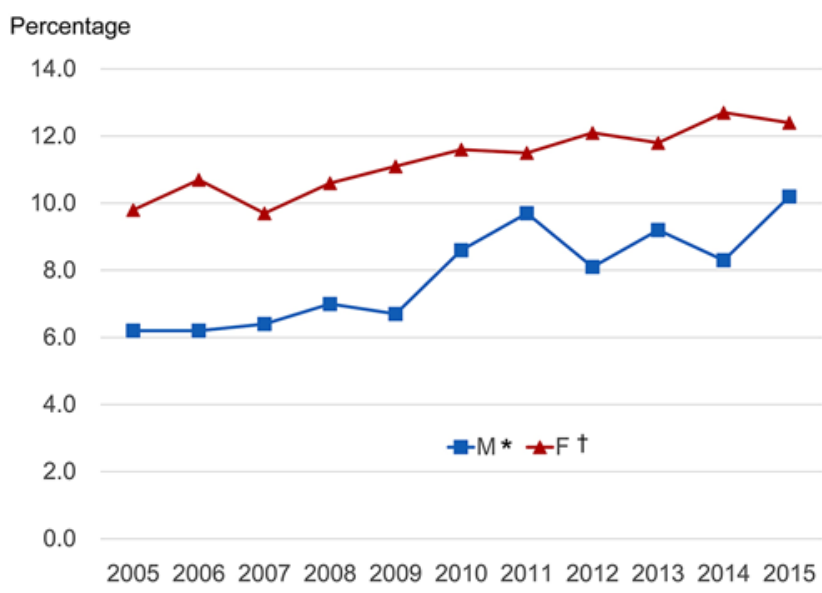

Year

FIG. 4. Annual trends in the age-standardized percentage of subjects taking cholesterol-lowering drugs. *Male $(p<0.0001)$; †female $(p=$ $0.0005)$. Figure is available in color online only.

through 2015. Accordingly, the decline in the incidence of SAH could relate to improvements in the general risk factors for SAH, such as hypertension and smoking, as was noted in a recent meta-analysis. ${ }^{8}$ While it is difficult to measure the direct impact of these health factors on the decline of SAH, we speculate that the significant decrease in SAH incidence may strongly relate to improved risk factor control. The present study revealed that we could not reduce the incidence of SAH through the radical treatment of individual UCAs because of the excessive numbers of UCAs. Reducing lifestyle diseases and the use of some drugs ${ }^{33,34}$ are possible solutions leading to the decline of SAH in Japan.

\section{Study Limitations}

To our knowledge, this is the first study to report the estimated nationwide SAH incidence rate and recent trends in SAH in Japan, but it does have several limitations. First, because of their retrospective register-based nature, the statistical data from the MHLW could not be precisely controlled. A population-based study or prospective observation study could be ideal for the SAH incidence rate, but it seemed impossible given the high cost. A register-based study can have coding errors or possibly inaccurate casefinding methods; however, it can also have a large number of cases. Therefore, we used a register-based study realistically. Second, the estimated nationwide age-standardized SAH incidence rate could not be directly searched because there has never been data on the nationwide SAH incidence in Japan. Therefore, deaths caused by SAH, as well as the population in the census, were used to estimate the nationwide age-standardized SAH incidence rate. Third, no reports have previously defined the formulas to estimate the SAH incidence rate or the estimated treatment rate of UCAs, and we could not know the change in SAH case-fatality rate and case-fatality rate after radical treatment of SAH during the research period; however, we assumed these rates according to previous studies. Fourth, because the Japanese population is rapidly aging, the age- 
standardized incidence rate also changes according to the year selected as the standard.

\section{Conclusions}

The nationwide SAH incidence rate in Japan is higher than in other countries. However, a recent decline trend in SAH was shown in this study. An improving lifestyle may have contributed to reducing the incidence of SAH in Japan. Further prospective studies are necessary to validate the reasons for this decline in SAH.

\section{Acknowledgments}

We thank Prof. Hiroki Ohkuma, MD, PhD; Dr. Tetsuji Inagawa, MD, PhD; and Dr. Nobuaki Michihata, $\mathrm{MD}, \mathrm{MPH}, \mathrm{PhD}$, for their guidance, and Julie A. Yamamoto, MA, for language and other editorial assistance.

This work was supported by the Pfizer Health Research Foundation Japan (19-E-01) and the Japan Society for the Promotion of Science Grant-in-Aid for Scientific Research (C) 17K10829.

\section{References}

1. Ingall T, Asplund K, Mähönen M, Bonita R. A multinational comparison of subarachnoid hemorrhage epidemiology in the WHO MONICA stroke study. Stroke. 2000;31(5):1054-1061.

2. Inagawa $T$. Trends in incidence and case fatality rates of aneurysmal subarachnoid hemorrhage in Izumo City, Japan, between 1980-1989 and 1990-1998. Stroke. 2001;32(7):14991507.

3. Korja M, Kaprio J. Controversies in epidemiology of intracranial aneurysms and SAH. Nat Rev Neurol. 2016;12(1):50-55.

4. de Rooij NK, Linn FH, van der Plas JA, et al. Incidence of subarachnoid haemorrhage: a systematic review with emphasis on region, age, gender and time trends. J Neurol Neurosurg Psychiatry. 2007;78(12):1365-1372.

5. Linn FH, Rinkel GJ, Algra A, van Gijn J. Incidence of subarachnoid hemorrhage: role of region, year, and rate of computed tomography: a meta-analysis. Stroke. 1996;27(4):625629.

6. van Gijn J, Kerr RS, Rinkel GJE. Subarachnoid haemorrhage. Lancet. 2007;369(9558):306-318.

7. Korja M, Lehto H, Juvela S, Kaprio J. Incidence of subarachnoid hemorrhage is decreasing together with decreasing smoking rates. Neurology. 2016;87(11):1118-1123.

8. Etminan N, Chang HS, Hackenberg K, et al. Worldwide incidence of aneurysmal subarachnoid hemorrhage according to region, time period, blood pressure, and smoking prevalence in the population: a systematic review and meta-analysis. JAMA Neurol. 2019;76(5):588-597.

9. Tanaka H, Ueda Y, Date C, et al. Incidence of stroke in Shibata, Japan: 1976-1978. Stroke. 1981;12(4):460-466.

10. Omama S, Yoshida Y, Ogasawara K, et al. Incidence rate of cerebrovascular diseases in northern Japan determined from the Iwate Stroke Registry with an inventory survey system. $J$ Stroke Cerebrovasc Dis. 2013;22(8):e317-e322.

11. Statistics of Japan Ministry of Health, Labour and Welfare [in Japanese]. https://www.e-stat.go.jp/ dbview?sid=0003214735. Accessed February 4, 2020.

12. Nakagawa T, Hashi K. The incidence and treatment of asymptomatic, unruptured cerebral aneurysms. J Neurosurg. 1994;80(2):217-223.

13. Ikawa F, Morita A, Tominari S, et al. Rupture risk of small unruptured cerebral aneurysms. J Neurosurg. 2019;1-10.

14. Morita A, Kirino T, Hashi K, et al. The natural course of unruptured cerebral aneurysms in a Japanese cohort. $N$ Engl J Med. 2012;366(26):2474-2482.
15. Greving JP, Wermer MJ, Brown RD Jr, et al. Development of the PHASES score for prediction of risk of rupture of intracranial aneurysms: a pooled analysis of six prospective cohort studies. Lancet Neurol. 2014;13(1):59-66.

16. Statistics Bureau of Japan. Population Census 2011. http:// www.stat.go.jp/english/data/kokusei/index.html. Accessed February 4, 2020.

17. Statistics of Japan Ministry of Health, Labour and Welfare. https://www.e-stat.go.jp/en/stat-search/ files?page $=1 \&$ kikan=00450. Accessed February 4, 2020.

18. Ministry of Health, Labour and Welfare. National Health and Nutrition Survey [in Japanese]. https://www.e-stat.go.jp/ stat-search/files?page $=1 \&$ layout=datalist $\&$ toukei $=00450171$ $\&$ tstat $=000001041744 \&$ cycle $=7 \&$ year $=20150 \&$ month $=0 \&$ tcl ass $1=000001098375$. February 4, 2020.

19. Organisation for Economic Co-operation and Development. Data, health, health equipment. https://data.oecd.org/health. htm\#profile-Health\%20equipment. Accessed February 4, 2020.

20. Ikeda N, Takimoto H, Imai S, et al. Data resource profile: the Japan National Health and Nutrition Survey (NHNS). Int J Epidemiol. 2015;44(6):1842-1849.

21. Murakami K, Livingstone MB, Okubo H, Sasaki S. Energy density of the diets of Japanese adults in relation to food and nutrient intake and general and abdominal obesity: a crosssectional analysis from the 2012 National Health and Nutrition Survey, Japan. Br J Nutr. 2017;117(1):161-169.

22. Alleyne $\mathrm{CH}$ Jr. Aneurysmal subarachnoid hemorrhage: have outcomes really improved? Neurology. 2010;74(19):14861487.

23. Hop JW, Rinkel GJ, Algra A, van Gijn J. Case-fatality rates and functional outcome after subarachnoid hemorrhage: a systematic review. Stroke. 1997;28(3):660-664.

24. Koffijberg H, Buskens E, Granath F, et al. Subarachnoid haemorrhage in Sweden 1987-2002: regional incidence and case fatality rates. J Neurol Neurosurg Psychiatry. 2008;79(3):294-299.

25. Sandvei MS, Mathiesen EB, Vatten LJ, et al. Incidence and mortality of aneurysmal subarachnoid hemorrhage in two Norwegian cohorts, 1984-2007. Neurology. 2011;77(20):1833-1839.

26. The-ACROSS-Group. Epidemiology of aneurysmal subarachnoid hemorrhage in Australia and New Zealand: incidence and case fatality from the Australasian Cooperative Research on Subarachnoid Hemorrhage Study (ACROSS). Stroke. 2000;31(8):1843-1850.

27. Hattori N, Katayama Y, Abe T. Case volume does not correlate with outcome after cerebral aneurysm clipping: a nationwide study in Japan. Neurol Med Chir (Tokyo). 2007;47(3):95-101.

28. Kurogi R, Kada A, Nishimura K, et al. Effect of treatment modality on in-hospital outcome in patients with subarachnoid hemorrhage: a nationwide study in Japan (J-ASPECT Study). J Neurosurg. 2018;128(5):1318-1326.

29. Vlak MH, Algra A, Brandenburg R, Rinkel GJ. Prevalence of unruptured intracranial aneurysms, with emphasis on sex, age, comorbidity, country, and time period: a systematic review and meta-analysis. Lancet Neurol. 2011;10(7):626-636.

30. Giordan E, Lanzino G, Rangel-Castilla L, et al. Risk of de novo aneurysm formation in patients with a prior diagnosis of ruptured or unruptured aneurysm: systematic review and meta-analysis. J Neurosurg. 2018;131(1):14-24.

31. Feigin VL, Rinkel GJ, Lawes CM, et al. Risk factors for subarachnoid hemorrhage: an updated systematic review of epidemiological studies. Stroke. 2005;36(12):2773-2780.

32. Sandvei MS, Romundstad PR, Müller TB, et al. Risk factors for aneurysmal subarachnoid hemorrhage in a prospective population study: the HUNT study in Norway. Stroke. 2009;40(6):1958-1962. 
33. Yoshimura Y, Murakami Y, Saitoh M, et al. Statin use and risk of cerebral aneurysm rupture: a hospital-based case-control study in Japan. J Stroke Cerebrovasc Dis. 2014;23(2):343-348.

34. Hudson JS, Marincovich AJ, Roa JA, et al. Aspirin and intracranial aneurysms. Stroke. 2019;50(9):2591-2596.

\section{Disclosures}

The authors report no conflict of interest concerning the materials or methods used in this study or the findings specified in this paper.

\section{Author Contributions}

Conception and design: Ikawa, Morita, Nakayama, Sakai, Iihara, Shiokawa, Date, Nozaki, Kinouchi, Houkin, Saito, Tominaga, Suzuki, Miyamoto, Kurisu, Arai. Acquisition of data: Ikawa, Morita, Nakayama, Sakai, Iihara, Shiokawa, Date, Nozaki, Kinouchi, Houkin, Saito, Tominaga, Suzuki, Miyamoto, Kurisu, Arai. Analysis and interpretation of data: Ikawa, Goto.

Drafting the article: Ikawa, Goto, Sakai, Ihara, Shiokawa, Date,
Kinouchi. Critically revising the article: Ikawa, Morita, Nozaki. Reviewed submitted version of manuscript: Ikawa, Morita,

Shiokawa, Nozaki, Tominaga. Approved the final version of the manuscript on behalf of all authors: Ikawa. Statistical analysis:

Ikawa, Nakayama, Goto. Administrative/technical/material support: Nozaki, Kinouchi, Houkin, Saito, Tominaga, Arai. Study supervision: Morita, Nakayama, Shiokawa, Date, Nozaki, Kinouchi, Saito, Tominaga, Suzuki, Miyamoto, Kurisu, Arai.

\section{Supplemental Information}

Online-Only Content

Supplemental material is available with the online version of the article.

Appendix. https://thejns.org/doi/suppl/10.3171/2020.1. JNS192848.

\section{Correspondence}

Fusao Ikawa: Graduate School of Biomedical and Health Sciences, Hiroshima University, Hiroshima, Japan. fikawa-nsu@umin.ac.jp. 